\title{
DECAY ANGULAR DISTRIBUTIONS OF INTERMEDIATE BOSONS PRODUCED BY PIONS AND BY COLLIDING BEAMS
}

\author{
H. UBERALL ${ }^{\dagger}$ \\ Harrison M. Randall Laboratory, University of Michigan, Ann Arbor, Michigan ${ }^{\dagger}$
}

Received 5 February 1964

\begin{abstract}
The leptonic decay angular distributions of intermediate bosons are obtained as a possible means of identifying the production reaction; for the latter, we considered the cases: a) W production by pions on protons (both two-body and inelastic reactions), treated in the peripheral (one-pion exchange) approximation and $b$ ) $\mathrm{W}$ pair production in colliding negaton-positon beam experiments. We also investigate the polarization state of the boson by obtaining the $3 \times 3$ density matrix, and note its connection with the decay angular distributions.
\end{abstract}

\section{Introduction}

Discovery of the intermediate boson ${ }^{1}$ ) W would be of essential importance for the theory of weak interactions. While at present, an experimental search is under way at CERN ${ }^{2}$ ) for bosons produced by neutrinos, other possible modes of production, e.g. by $\mu$ mesons ${ }^{3,4}$ ), $\pi$ mesons ${ }^{5}$ ), photons ${ }^{6-8}$ ) and colliding negaton-positon beams ${ }^{9}$ ) should thus be studied also. Since the background problems in these reactions will be considerable, it seems desirable to know differential cross sections of the $\mathrm{W}$ decay products besides the total $\mathrm{W}$ production rates, in order to plan experiments or to identify the production reaction in question. We here present the angular dist ibutions of charged leptons - the decay of $\mathrm{W}$ into a lepton and a neutrino, with the now likely ${ }^{2}$ ) boson mass of $M \approx 1.3 \mathrm{~m}_{\mathrm{p}}$, will be a dominant decay mode ${ }^{10}$ )from the decay of the intermediate boson subsequent to its production in the following reactions:

(a) $\mathrm{W}$ production by pions on protons,

$$
\begin{gathered}
\pi^{ \pm}+\mathrm{p} \rightarrow \mathrm{W}^{ \pm}+\mathrm{p}, \\
\pi^{ \pm}+\mathrm{p} \rightarrow \mathrm{W}^{ \pm}+\text {all others; }
\end{gathered}
$$

(b) W pair production in colliding negaton-positon beams,

$$
\mathrm{e}^{-}+\mathrm{e}^{+} \rightarrow \mathrm{W}^{+}+\mathrm{W}^{-} \text {. }
$$

In sect. 2 we discuss results for reactions (1) and (2) obtained in the peripheral (onepion exchange) approximation, which is expected to be valid at least qualitatively; total cross sections are shown, and it is found from the $3 \times 3$ density matrix of the

$t$ Present address: Department of Physics, The Catholic University of America, Washington D.C.

t† Work supported in part by the Office of Naval Research, U.S. Navy. 
W that it carries predominantly a longitudinal linear polarization; the charged decay lepton angular distribution relative to the incident pion is governed by this polarization and was obtained by folding the $4 \times 4$ density matrix of production into that of decay, in a fashion described in ref. ${ }^{4}$ ). In sect. 3 , total cross sections, the density matrix and decay angular distributions with respect to the incoming electron beam are discussed for reaction (3), for a $\mathrm{W}$ assumed to carry both anomalous magnetic moment and electric quadrupole moment.

\section{Production by Pions}

Fig. 1 presents the peripheral diagrams used by us to describe reactions (1) and (2) and gives the assignment of variables. The matrix element of (1) has been found ${ }^{5}$ )

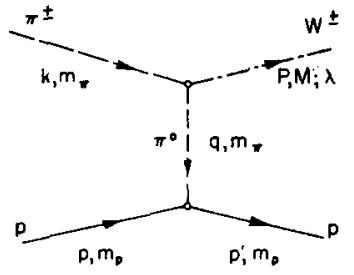

(a)

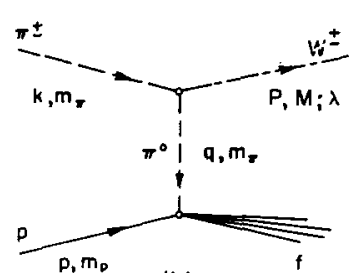

(b)

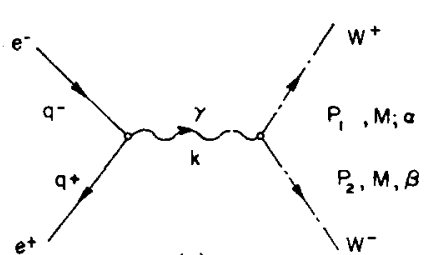

(c)

Fig. 1. Feynman diagrams used for calculation of reactions(1)-(3).

from the conserved vector current hypothesis as

$$
M_{1}=\sqrt{2} g F_{\pi}\left(M^{2}\right) \frac{\varepsilon_{\lambda}^{*}(k+q)_{\lambda}}{\left(4 k E_{\mathrm{p}}\right)^{\frac{2}{2}}} \frac{g_{\pi \mathrm{N}} f_{\mathrm{NNN}}\left(q^{2}\right)}{q^{2}+m_{\mathrm{\pi}}^{2}} \bar{u}_{\mathrm{p}^{\prime}} \gamma_{5} u_{\mathrm{p}},
$$

where $g / M=2^{-\frac{1}{2}} G_{\mathrm{V}}^{\frac{1}{\mathrm{t}}}, g_{\pi N}^{2} / 4 \pi \approx 15$, the vector $\beta$-decay coupling constant $G_{\mathrm{V}}=10^{-5}$ $m_{\mathrm{p}}^{-2}, m_{\mathrm{p}}$ being the proton mass, $F_{n}$ is the electromagnetic pion form factor, $f_{n N \mathrm{~N}}$ the pion-nucleon vertex form factor and $\varepsilon_{\lambda}$ the W polarization four-vector; $F_{\mathrm{x}}\left(M^{2}\right)$ is of order 1 since $M \approx 1.3 m_{\mathrm{p}}$ is far from the resonance ${ }^{5}$ ). For the total cross section, we obtain from eq. (4)

$$
\frac{\sigma_{1}}{\left|F_{\pi}\left(M^{2}\right)\right|^{2}}=\frac{g^{2}}{32 \pi} \frac{g_{\pi \mathrm{N}}^{2}}{\left(k m_{\mathrm{p}} M\right)^{2}} \int_{\Delta-^{2}}^{\Delta+^{2}}\left|f_{\pi N \mathrm{~N}}\left(\Delta^{2}\right)\right|^{2}\left(\frac{\Delta^{2}+M^{2}}{\Delta^{2}+m_{\pi}^{2}}\right)^{2} \Delta^{2} \mathrm{~d} \Delta^{2}
$$

Similarly, the matrix element of (2) is

$$
M_{2}=\sqrt{2} g F_{\mathrm{x}}\left(M^{2}\right) \frac{\varepsilon_{\lambda}^{*}(k+q)_{\lambda}}{\left(4 k E_{\mathrm{P}}\right)^{\frac{1}{2}}} \frac{\left(2 E_{q}\right)^{\frac{1}{2}}}{q^{2}+m_{\pi}^{2}} A\left(\pi^{0}+\mathrm{p} \rightarrow \mathrm{f}\right),
$$

in terms of the total scattering amplitude $A$ of a real $\pi^{0}$ on a proton; it gives a cross 
section

$$
\begin{array}{r}
\frac{\sigma_{2}}{\left|F_{\pi}\left(M^{2}\right)\right|^{2}}=\frac{g^{2}}{8 \pi^{2} k^{2} M^{2} m_{\mathrm{p}}} \int_{m_{\mathrm{p}}+m_{\pi}}^{\Omega-M} W \mathrm{~d} W \sigma_{\pi^{0}+\mathrm{p} \rightarrow \mathrm{f}}^{\mathrm{tot}}(W) \int_{\Delta_{-}^{2}}^{\Delta+^{2}} \mathrm{~d} \Delta^{2}\left|f_{\pi \mathrm{NN}}\left(\Delta^{2}\right)\right|^{2}\left(E_{q}^{2}+\Delta^{2}\right)^{\frac{1}{2}} \\
\times\left(\frac{\Delta^{2}+M^{2}}{\Delta^{2}+m_{\pi}^{2}}\right)^{2},
\end{array}
$$

in terms of the total $\pi^{0}-\mathrm{p}$ scattering cross section, which from isobaric spin considerations becomes just

$$
\sigma_{x^{0} p}=\frac{1}{2}\left(\sigma_{x^{+} p}+\sigma_{x-p}\right),
$$

and the total $\pi^{ \pm}$p cross sections can be found in the literature. The argument is taken as $W$, the total energy of the particles $f$ in their c.m. system. In the laboratory, the total energy in the overall c..m system becomes

$$
\Omega=\left[m_{\mathrm{p}}\left(m_{\mathrm{p}}+2 k\right)\right]^{\frac{1}{2}} .
$$

The limits on the $\Delta^{2}$ integration are

with

$$
\Delta_{ \pm}^{2}=2 k^{*}\left[E_{\mathrm{P}}^{*} \pm\left(E_{\mathrm{P}}^{* 2}-M^{2}\right)^{\frac{1}{2}}\right]
$$

$$
E_{\mathrm{p}}^{*}=\left(\Omega^{2}+M^{2}-W^{2}\right) / 2 \Omega, \quad k^{*}=\left(\Omega^{2}-m_{\mathrm{p}}^{2}\right) / 2 \Omega .
$$

In eq. (5) these limits are to be used with $W$ replaced by $m_{p}$. For the pion-nucleon form factor, we took

$$
f \pi_{\mathrm{NN}}\left(q^{2}\right) \approx\left(1+q^{2} / \alpha\right)^{-1}
$$

with $\alpha \approx 50 m_{\pi}^{2}$, as suggested from peripheral calculations of Ferrari and Selleri ${ }^{11}$ ) on nucleon-nucleon scattering. (Drell and Hiida ${ }^{12}$ ) chose for the same process a square cutoff at $\Delta^{2} \approx 16 \mathrm{~m}_{\pi}^{2}$ ). The factor $\left|f_{\pi N N}\left(\Delta^{2}\right)\right|^{2}$ was also used in eq. (7) at the lower vertex. Results are presented in fig. 2(a) (and the effect of changing $\alpha$ in reaction (1) is indicated). We see that near threshold the two-body reaction dominates, even though the $\gamma_{5}$ interaction adds an extra $\Delta^{2}$ factor, which is usually believed ${ }^{13}$ ) to decrease the two-body process considerably relative to the inelastic one. In our case, however, the inelastic process will dominate only at higher energies.

The difficulty to distinguish reactions (1) and (2) in the background of the strong interactions has been stated before, and special methods, such as observation of the recoil proton ${ }^{5}$ ) in reaction (1), in particular ${ }^{1}$ ) together with the leptonic decay of the $W$, have been suggested. We here obtain the angular distribution of the charged lepton from

$$
\mathrm{W}^{ \pm} \rightarrow 1^{ \pm}+v
$$

with respect to the incident pion, the directions of the unobserved $\mathrm{W}$ being summed over, which will be useful in the design of experiments trying to detect reactions (1) and (2). Since the $1^{ \pm}$angular distribution depends on the $W$ polarization ${ }^{4}$ ), we shall first consider the latter, as described by its density matrix. If the differential production 
cross section $d \sigma$, summed over unobserved variables but not the $W$ spin, is written in the form

$$
\mathrm{d} \sigma=\varepsilon_{\alpha}^{*} \varepsilon_{\beta} Q_{\alpha \beta},
$$

the density matrix of the $W$ in the corresponding production reaction is

$$
\rho_{\alpha \beta}=\left(\operatorname{Tr} Q+M^{-2} P_{\lambda} P_{\mu} Q_{\lambda \mu}\right)^{-1}\left(\delta_{\alpha \rho}+M^{-2} P_{\alpha} P_{\rho}\right) Q_{\rho \sigma}\left(\delta_{\sigma \beta}+M^{-2} P_{\sigma} P_{\beta}\right),
$$

or in $3 \times 3$ form (with respect to a basis $\varepsilon^{(i)}, i=123$ ):

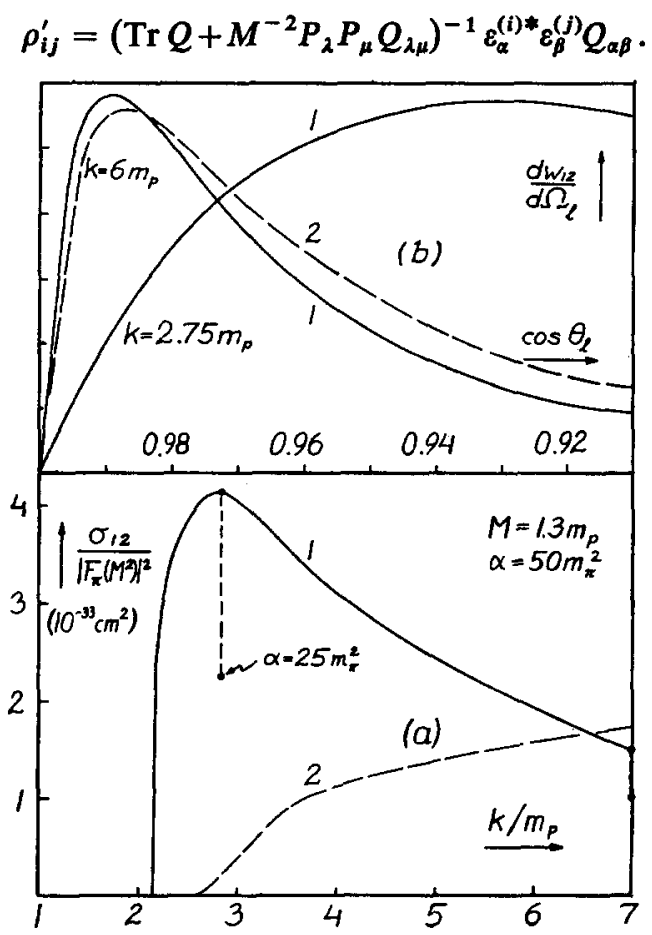

Fig. 2. $\mathrm{W}$ production cross section (a) and laboratory angular distributions of charged $\mathrm{W}$ decay leptons (b) for reactions (1) and (2).

We obtained the $3 \times 3$ density matrix in completely differential form for both the reactions (1) and (2) as

$\rho^{\prime}=\left(E_{\mathrm{P}}-P \cos \theta\right)^{-2}\left(\begin{array}{lll}0 & 0 & 0 \\ 0 & M^{2} \sin ^{2} \theta & M\left(E_{\mathrm{P}} \cos \theta-P\right) \sin \theta \\ 0 & M\left(E_{\mathrm{P}} \cos \theta-P\right) \sin \theta & \left(E_{\mathrm{P}} \cos \theta-P\right)^{2}\end{array}\right)$,

where $\theta=\Varangle(\boldsymbol{k}, \boldsymbol{P})$. For $E_{\mathrm{P}} / M \rightarrow \infty$ and for $\cos \theta \rightarrow \pm 1$, this gives

$$
\rho_{i j}^{\prime} \rightarrow \delta_{i 3} \delta_{j 3}=\varepsilon^{(i) *} \cdot \varepsilon^{(3)} \varepsilon^{(3) *} \cdot \varepsilon^{(j)},
$$

i.e. the $\mathrm{W}$ has $100 \%$ linear longitudinal polarization. (We tooke a right-handed 
linear basis $\varepsilon^{(i)}$ with $\varepsilon^{(3)}\left\|P, \varepsilon^{(1)}\right\| k \times P$.) In the decay (13), the leptons come out ${ }^{4}$ ) predominantly normal to the linear boson polarization direction in the $\mathrm{W}$ rest frame; for decay in flight, this maximum will be shifted to small but finite forward angles.

If $\rho$ is folded into the density matrix of the $W$ in the decay (13) (eq. (80) of ref. ${ }^{4}$ )) and the intermediate $W$ directions integrated out, we obtain the laboratory $1^{ \pm}$angular distributions shown in fig. 2(b), where $\theta_{l}$ is the angle between the directions $k$ of the incident pion and $l$ of the decay lepton. This shows the similarity of the angular distributions in reactions (1) and (2) and confirms the qualitative statements made above. Near threshold, the $\mathrm{W}$ is slow and does not always go forward, hence a broad angular distribution of $\mathrm{l}^{ \pm}$. The scale of this figure is arbitrary.

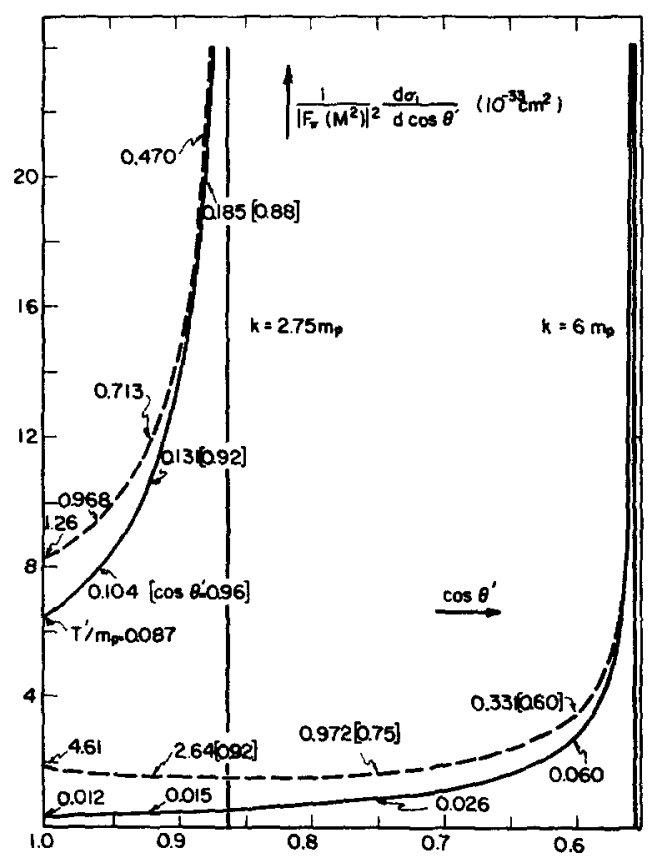

Fig. 3. Recoil proton laboratory angular distribution (and recoil kinetic energies) for reaction (1).

The foregoing results are of interest in a chamber-type experiment. If, as suggested by Lee and Yang ${ }^{1}$ ), the recoil proton momentum is measured (together with the $\mathrm{W}$ decay lepton momentum) in a counter experiment on reaction (1), one needs the laboratory angular distribution of the recoil protons

$\frac{1}{\left|F_{\pi}\left(M^{2}\right)\right|^{2}} \frac{\mathrm{d} \sigma_{1}}{\mathrm{~d} \cos \theta^{\prime}}=\frac{g^{2}}{4 M^{2}} \frac{g_{\pi \mathrm{N}}^{2}}{4 \pi}\left|f_{\pi \mathrm{NN}}\left(q^{2}\right)\right|^{2} \frac{q^{2}}{k m}\left(\frac{q^{2}+M^{2}}{q^{2}+m_{\pi}^{2}}\right)^{2} \frac{p^{\prime 2}}{\left|p^{\prime}(k+m)-E^{\prime} k \cos \theta^{\prime}\right|}$,

where $\theta^{\prime} \Varangle\left(\boldsymbol{p}^{\prime}, \boldsymbol{k}\right)$, shown in fig. 3. In the laboratory, up to a maximum angle, there are two groups of protons with different energies emerging at a given angle, hence the 
two curves; at certain angles, recoil proton kinetic energies $T^{\prime}$ have been indicated in the figure. The integrable infinity is of kinematic origin.

A fixed recoil proton momentum also determines the direction of the produced $W$. Suppose its lepton decay angular distribution in the laboratory is measured with respect to this direction as the $z$-axis, with the polar angle $\Theta=\Varangle(P, l)$ and the azimuth $\Phi=\Varangle$ (Pk plane, $\boldsymbol{P l}$ plane) (on which the angular distribution will depend also, since the $\mathrm{W}$ is not purely longitudinally polarized, see eq. (17)). For each of the two aforementioned proton groups of different energies separately, the angular distributions (from folding of production and decay density matrices) are in this differential case

$$
\frac{\mathrm{d} w_{1}}{\mathrm{~d} \Omega_{l}} \propto\left(1-\cos \theta_{l}\right) \frac{E_{\mathrm{P}}\left(q^{2}+M^{2}\right) z^{3}-k M^{2}\left(1-\cos \theta_{l}\right) z^{4}}{E_{\mathrm{P}}^{5}\left(q^{2}+M^{2}\right)^{2}},
$$

(which is seen to be independent of the form factor), with

$$
\begin{gathered}
z=E_{\mathrm{P}}\left(E_{\mathrm{P}}-P \cos \theta\right)^{-1}, \\
\cos \theta_{l}=\cos \theta \cos \Theta+\sin \theta \sin \theta \cos \Phi .
\end{gathered}
$$

Figs. 4 and 5 present these angular distributions on an arbitrary scale plotted versus $\cos \Theta$, with $\Phi$ as parameter, for incident energy $k=2.75 m_{p}$ and proton recoil

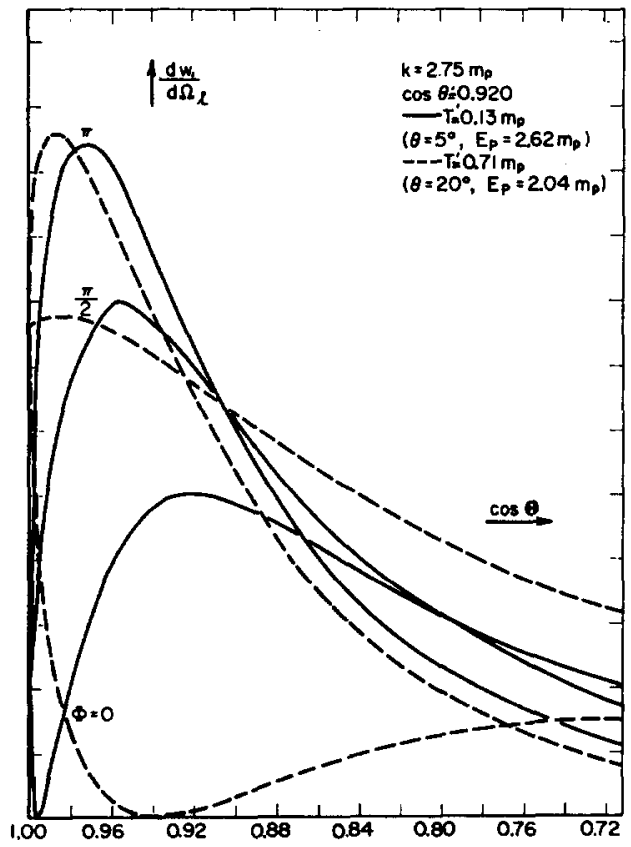

Fig. 4. Laboratory angular distributions of charged lepton from $W$ decay of flight in reaction (1), at recoil proton angle $\cos \theta^{\prime}=\mathbf{0 . 9 2 0}$.

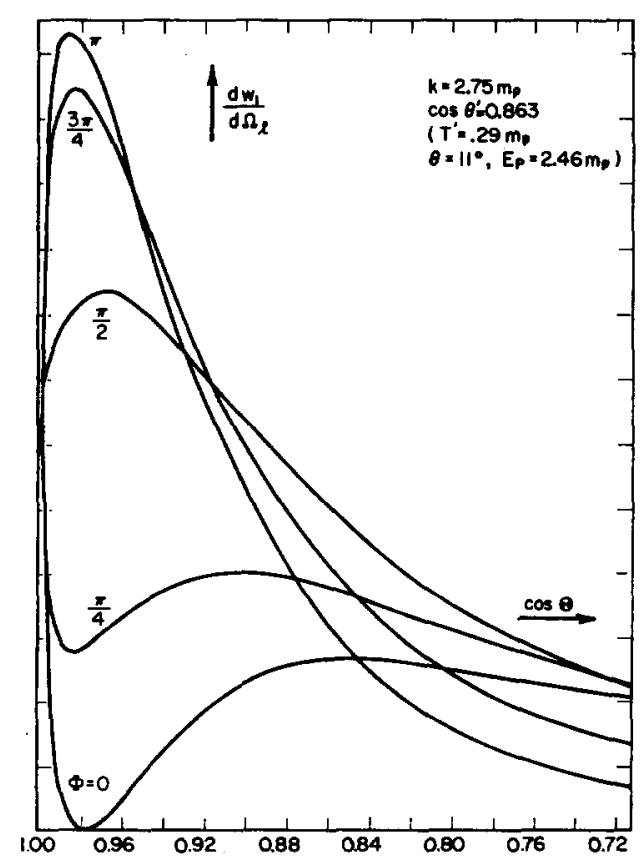

Fig. 5. Laboratory angular distributions of charged lepton from $W$ decay in flight in reaction (1), at maximum recoil proton angle $\cos \theta^{\prime}=\mathbf{0 . 8 6 3}$. 
directions $\cos \theta^{\prime}=0.920$ and $\cos \theta^{\prime}=0.863$; the latter value corresponds to the maximum recoil angle, where also the two $T^{\prime}$ coincide. At $k=6 m_{\mathrm{p}}$, the decay lepton angular distributions are found to be much less dependent on $\Phi$ (since here, the W polarization becomes more complete and forward), and are peaked around $\cos \Theta=$ $0.98-0.99$, but qualitatively similar to those of figs. 4 and 5 .

\section{Production in Colliding $\mathrm{e}^{-}-\mathrm{e}^{+}$Beams}

Fig. 1 also presents the relevant Feynman diagram and assignment of momenta for this process. The laboratory and c.m. systems coincide here, and each of the four particles has the same energy $E$. In lowest order in e, the electromagnetic interaction Lagrangian of the $\mathrm{W}$ carrying an anomolous magnetic moment $\boldsymbol{\kappa}$ and anomalous electric quadrupole moment $\gamma$ is given by ${ }^{14}$ )

$$
\begin{aligned}
\mathscr{L}_{\mathrm{int}}=-\frac{1}{2} i e\left(A_{\mu} \varphi_{\nu}^{*}-A_{\nu} \varphi_{\mu}^{*}\right) & \left(\partial_{\mu} \varphi_{\nu}-\partial_{\nu} \varphi_{\mu}\right)+\frac{1}{2} i e\left(\partial_{\mu} \varphi_{\nu}^{*}\right. \\
& \left.-\partial_{\nu} \varphi_{\mu}^{*}\right)\left(A_{\mu} \varphi_{\nu}-A_{\nu} \varphi_{\mu}\right)-i e \kappa F_{\mu \nu} \varphi_{\mu}^{*} \varphi_{\nu} \\
& +\frac{i e \gamma}{4 M^{2}}\left[\left(\partial_{\mu} \varphi_{\nu}^{*}-\partial_{\nu} \varphi_{\mu}^{*}\right) \varphi_{\lambda}-\varphi_{\lambda}^{*}\left(\partial_{\mu} \varphi_{\nu}-\partial_{\nu} \varphi_{\mu}\right)\right] \partial_{\lambda} F_{\mu \nu}
\end{aligned}
$$

it was shown by Lee and Yang ${ }^{15}$ ) for the case $\gamma=0$ that in lowest order, the vertex may be taken as $-\mathscr{L}_{\text {int }}$, even though eq. (21) contains field derivatives. For $\gamma \neq 0$, the theory is considerd non-renormalizable and not positive definite. In spite of this, Cabibbo and Gatto ${ }^{9}$ ) have derived in lowest order the most general form of the electromagnetic boson vertex in Fig. 1c from Lorentz, gauge, and charge conjugation invariance and found that it comes out exactly as derived from a Lagrangian theory, and by setting the Hamiltonian equal to $-\mathscr{L}_{\text {int }}^{\prime}$, where

$$
\begin{gathered}
\mathscr{L}_{\mathrm{int}}^{\prime}=\left(j_{\mu}+j_{\mu}^{\prime}+j_{\mu}^{\prime \prime}\right) A_{\mu}, \\
j_{\mu}=-i e\left[\left(\partial_{\mu} \varphi_{\nu}-\partial_{\nu} \varphi_{\mu}\right) \varphi_{\nu}^{*}-\left(\partial_{\mu} \varphi_{v}^{*}-\partial_{\nu} \varphi_{\mu}^{*}\right) \varphi_{v}\right], \\
j_{\mu}^{\prime}=-i e \kappa \partial_{\nu}\left(\varphi_{\mu}^{*} \varphi_{\nu}-\varphi_{\nu}^{*} \varphi_{\mu}\right), \\
j_{\mu}^{\prime \prime}=\frac{i e \gamma}{2 M^{2}} \partial_{v}\left[\left(\partial_{\mu} \varphi_{\lambda}^{*}-\partial_{\lambda} \varphi_{\mu}^{*}\right)\left(\partial_{v} \varphi_{\lambda}-\partial_{\lambda} \varphi_{v}\right)-\left(\partial_{v} \varphi_{\lambda}^{*}\right.\right. \\
\left.\left.-\partial_{\lambda} \varphi_{v}^{*}\right)\left(\partial_{\mu} \varphi_{\lambda}-\partial_{\lambda} \varphi_{\mu}\right)+M^{2}\left(\varphi_{\mu}^{*} \varphi_{\nu}-\varphi_{v}^{*} \varphi_{\mu}\right)\right]
\end{gathered}
$$

the equivalence of (22) and (23) with eq. (21) may be demonstrated to lowest order in e. Eq. (22) leads to a matrix element ${ }^{9}$ )

$$
\begin{gathered}
M_{3}=\bar{v}_{q}+\gamma_{\mu} u_{q}-\mathfrak{M}_{\mu}, \\
\mathfrak{M}_{\mu}=\frac{e^{2}}{2 E k^{2}} \varepsilon_{1 \alpha}^{*} \varepsilon_{2 \beta}^{*}\left[\delta_{\alpha \beta} K_{\mu}+(1+\kappa)\left(k_{\alpha} \delta_{\beta \mu}-k_{\beta} \delta_{\alpha \mu}\right)+\frac{\gamma}{2 M^{2}}\left(k_{\alpha} k_{\beta}-\frac{1}{2} k^{2} \delta_{\alpha \beta}\right) K_{\mu}\right],
\end{gathered}
$$


where $K=P_{1}-P_{2}$, a cross section

$$
\frac{\mathrm{d} \sigma_{3}}{\mathrm{~d} \Omega_{1}}=\frac{E^{2} \beta}{32 \pi^{2}} \sum_{\mathrm{pol}}\left(\mathfrak{M}^{*} \cdot \mathfrak{M}-E^{-2} \mathfrak{M}^{*} \cdot q^{-\mathfrak{M} \cdot q^{-}}\right),
$$

where $\beta=\left(1-x^{-2}\right)^{\frac{1}{2}}, x=E / M$, and finally, analogously to eq. (15), a density matrix ${ }^{9}$ ) (we here use $\sigma_{\lambda \mu}$ such that

$$
\mathrm{d} \sigma_{3}=\sum_{\mathrm{pol} 1} \varepsilon_{1 \lambda}^{*} \varepsilon_{1 \mu} \sigma_{\lambda \mu}
$$

actually, $\rho$ has to be normalized to unit trace):

$$
\begin{aligned}
\rho_{\lambda \mu} \sim \sigma_{\lambda \mu} & =\frac{\alpha^{2} \beta \mathrm{d} \Omega_{1}}{2^{7} E^{2} M^{2}}\left[M^{2} B_{1} \delta_{\lambda \mu}+B_{2} k_{\lambda} k_{\mu}+B_{3} Q_{\lambda} Q_{\mu}\right. \\
& \left.+B_{4} K_{\lambda} K_{\mu}-B_{5}\left(k_{\lambda} Q_{\mu}+k_{\mu} Q_{\lambda}\right)+B_{6}\left(k_{\lambda} K_{\mu}+k_{\mu} K_{\lambda}\right)-B_{7}\left(K_{\lambda} Q_{\mu}+K_{\mu} Q_{\lambda}\right)\right],
\end{aligned}
$$

where $k=P_{1}+P_{2}, Q=q^{-}-q^{+}, \alpha=1 / 137$, and where (correcting the ambiguity of some unpaired brackets in ref. $\left.{ }^{9}\right)$ ):

$$
\begin{aligned}
& B_{1}=4 \beta^{2} \sin ^{2} \theta\left(1+x^{2} \gamma\right)^{2}+4 x^{2} \beta^{2}(1+\kappa)^{2}, \\
& B_{2}=x^{2} \beta^{2}\left(1+\beta^{2} \cos ^{2} \theta\right)(1+\kappa)^{2}+\beta^{2} \sin ^{2} \theta\left\{\left[1+x^{2}(2 \kappa-\gamma)\right]^{2}+4\left(1+x^{2} \kappa\right)(\gamma-\kappa)\right\}, \\
& B_{3}=-\beta^{2}(1+\kappa)^{2}, \\
& B_{4}=x^{2} \beta^{2}\left(1+\cos ^{2} \theta\right)(1+\kappa)^{2}+4 x^{2}(1+\kappa)(\kappa-\gamma)+\beta^{2} \sin ^{2} \theta\left[1+2 x^{2} \gamma+x^{4}(2 \kappa-\gamma)^{2}\right], \\
& B_{5}=2 \beta^{3} x^{2} \cos \theta(1+\kappa)(\kappa-\gamma), \\
& B_{6}=x^{2} \beta^{2}\left(1+\cos ^{2} \theta\right)(1+\kappa)^{2}+\beta^{2} \sin ^{2} \theta\left[1+x^{2}(2 \kappa-\gamma)\right]^{2}+2 x^{2} \beta^{2} \cos ^{2} \theta(1+\kappa)(\kappa-\gamma), \\
& B_{7}=-\beta \cos \theta(1+\kappa)^{2}+2 \beta x^{2} \cos \theta(1+\kappa)(\kappa-\gamma),
\end{aligned}
$$

with $\theta=\nless\left(\boldsymbol{P}_{1}, \boldsymbol{q}^{-}\right)$. We have set the form factors introduced in ref. $\left.{ }^{9}\right)$ equal to unity, and the summation over the polarizations of the $W$ with polarization vector $\varepsilon_{2}$ has been carried out, since we do not consider polarization correlations. The cross section following from eq. (27)

$$
\frac{\mathrm{d} \sigma_{3}}{\mathrm{~d} \cos \theta}=\frac{\pi \alpha^{2} \beta^{3}}{16 E^{2}}\left\{2 x^{2}(1+\kappa)^{2}\left(1+\cos ^{2} \theta\right)+\sin ^{2} \theta\left[2\left(1+x^{2} \gamma\right)^{2}+\left(1+x^{2}(2 \kappa-\gamma)\right)^{2}\right]\right\}
$$

and integrated over $\cos \theta$, is shown in fig. 6 for $M=1.3 m_{\mathrm{p}}$. It depends essentially on both $\kappa$ and $\gamma$. Without form factors, unitarity will be violated, as remarked by Cabibbo and Gatto: for $\kappa=\gamma=0$ at energies of the order $100 \mathrm{GeV}$; for $\kappa \neq 0$ or $\gamma \neq 0$, at some lower energy. Using a right-handed reference system $\varepsilon^{(3)} \| P_{1}$, $\boldsymbol{\varepsilon}^{(1)} \| \boldsymbol{q}^{-} \times \boldsymbol{P}_{1}$, eq. (28) gives the $3 \times 3$ density matrix 


$$
\begin{aligned}
& \rho^{\prime}=\frac{1}{4} \beta^{-2}\left\{2 x^{2}(1+\kappa)^{2}\left(1+\cos ^{2} \theta\right)+\sin ^{2} \theta\left[2\left(1+x^{2} \gamma\right)^{2}+\left(1+x^{2}(2 \kappa-\gamma)\right)^{2}\right]\right\}^{-1} \\
& \times\left(\begin{array}{lc}
B_{1}, 0, & 0 \\
0, B_{1}+4 x^{2} B_{3} \sin ^{2} \theta, & 4 x^{3} \sin \theta\left[B_{3} \cos \theta+\beta\left(B_{5}-B_{7}\right)\right] \\
0,4 x^{3} \sin \theta\left[B_{3} \cos \theta+\beta\left(B_{5}-B_{7}\right)\right], & B_{1}+4 x^{4}\left[\beta^{2}\left(B_{2}+B_{4}\right)+B_{3} \cos ^{2} \theta\right. \\
& \left.+2 \beta\left(\left(B_{5}-B_{7}\right) \cos \theta-\beta B_{6}\right)\right]
\end{array}\right)
\end{aligned}
$$

which for $\kappa=\gamma=0$ simplifies to

$$
\begin{aligned}
\rho^{\prime}=\left[2 x^{2}\left(1+\cos ^{2} \theta\right)+3 \sin ^{2} \theta\right]^{-1} & \\
& \times\left(\begin{array}{lll}
x^{2}+\sin ^{2} \theta & 0 & 0 \\
0 & x^{2} \cos ^{2} \theta+\sin ^{2} \theta & 0 \\
0 & 0 & x^{2}\left(1+\cos ^{2} \theta\right)+\sin ^{2} \theta
\end{array}\right) .
\end{aligned}
$$

This is an incoherent mixture of pure-state density matrices

$$
\rho_{i j}^{(m)^{\prime}}=\delta_{i m} \delta_{j m}, \quad m=1,2,3,
$$

corresponding to linear polarizations in the transverse directions $m=1$ and 2 and in the longitudinal direction 3. Since here, unlike in sect. 2, kinematics does not

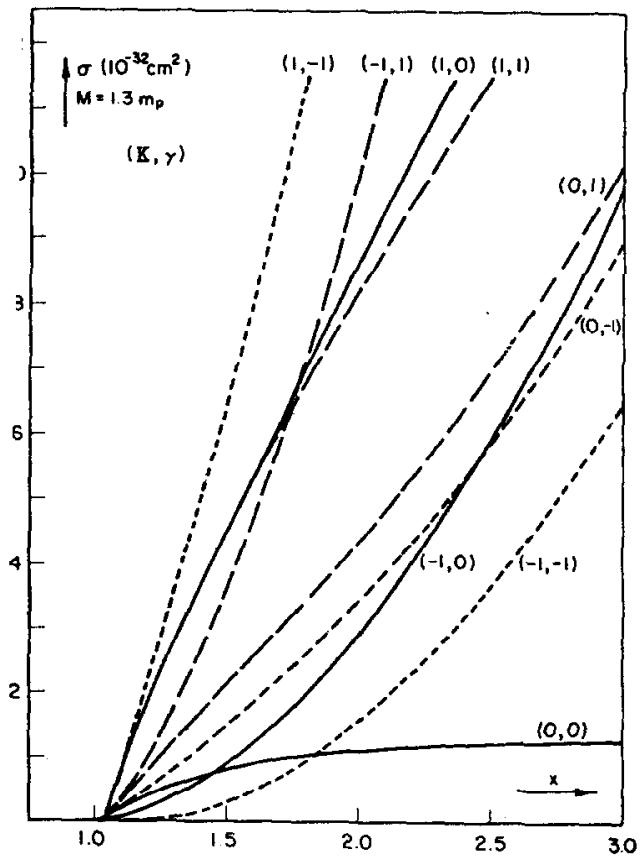

Fig. 6. $W$ pair production cross sections in a colliding electron-positon beam experiment, for various values of anomalous magnetic moment and electric quadrupole moment of the boson.

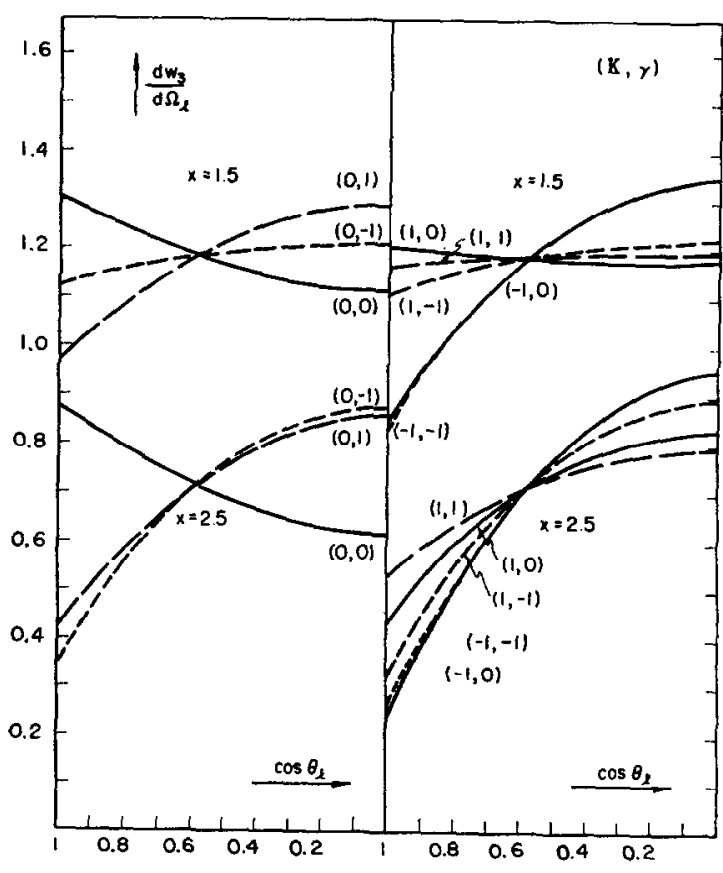

Fig. 7. Angular distributions of charged leptons from the decay of $\mathrm{W}$ produced in reaction (3). 
collimate the produced $W$, no easy prediction can be made from its polarization state to the lepton decay angular distribution. The latter is again obtained by folding the density matrix of eq. (28) into that of decay, and again we must integrate out the directions of the intermediate boson since it remains unobservable, with the result

$$
\begin{aligned}
& \frac{\mathrm{d} w_{3}}{\mathrm{~d} \Omega_{l}} \propto \beta^{-2} x^{-3}\left[4 x^{2}(1+\kappa)^{2}+2\left(1+x^{2} \gamma\right)^{2}+\left(1+x^{2}(2 \kappa-\gamma)\right)^{2}\right]^{-1} \\
& \times \int_{-1}^{1} \mathrm{~d} \cos \theta\left\{B_{1} J_{2}-2\left[x^{2}\left(J_{2}-2 J_{3}\right)+J_{4}\right]\left(B_{2}+B_{4}-2 B_{6}\right)\right. \\
&+2\left[x^{2}\left(J_{2}+2 \beta \cos \theta \cos \theta_{l} J_{3}\right)-\cos ^{2} \theta_{l} J_{4}\right] B_{3} \\
&\left.+4\left[x^{2}\left(\beta \cos \theta+\cos \theta_{l}\right) J_{3}-\cos \theta_{l} J_{4}\right]\left(B_{5}-B_{7}\right)\right\},
\end{aligned}
$$

where $\theta_{l}=\Varangle\left(q^{-}\right.$, charged lepton from $\mathrm{W}$ decay $)$, and

$$
\begin{aligned}
& J_{2}=\left(1-\beta \cos \theta \cos \theta_{l}\right) X^{-\frac{7}{2}}, \\
& J_{3}=\left[\left(1-\beta \cos \theta \cos \theta_{l}\right)^{2}+\frac{1}{2} \beta^{2} \sin ^{2} \theta \sin ^{2} \theta_{l}\right] X^{-\frac{1}{l}}, \\
& J_{4}=\left(1-\beta \cos \theta \cos \theta_{l}\right)\left[\left(1-\beta \cos \theta \cos \theta_{l}\right)^{2}+\frac{3}{2} \beta^{2} \sin ^{2} \theta \sin ^{2} \theta_{l}\right] X^{-7}, \\
& X=\left(1-\beta \cos \theta \cos \theta_{l}\right)^{2}-\beta^{2} \sin ^{2} \theta \sin ^{2} \theta_{l} .
\end{aligned}
$$

These angular distributions are shown in fig. 7 at two energies and for various values of $\kappa$ and $\gamma$, upon which they are seen to depend. The distributions are symmetric about $\cos \theta_{l}=0$. The scale of the figure is arbitrary.

\section{Conclusion}

We obtained angular distributions of charged leptons from the decay of intermediate bosons produced in pion-proton reactions, or in $\mathrm{e}^{-} \mathrm{e}^{+}$colliding beam experiments, with the boson itself being considered unobserved. These differential cross sections represent useful information beyond that given by the total cross sections, and it is only they that have experimental meaning (rather than, e.g., the differential cross section of eq. (30)), since the short-lived W cannot be observed but through its decay products. Density matrices are also cast into a $3 \times 3$ form, in order to give information about the polarization state of the produced intermediate boson.

I wish to thank Professor R. C. F. Bartels for making the facilities of the University of Michigan Computing Center available for this work, and Mrs. Dolores Moebs for help with the numerical computation. I am also grateful to Mr. Howard Saxer for providing me with a compilation of total pion-proton cross sections. An enlightening discussion with Dr. A. L. Read on experimental aspects of these problems is appreciated. 


\section{References}

1) T. D. Lee and C. N. Yang, Phys. Rev. 119 (1960) 1410

2) Report to the Conference on Weak Interactions, Brookhaven National Laboratory, Sept. 1963.

3) M. E. Ebel and W. D. Walker, Phys. Rev. 122 (1961) 1639

4) H. Uberall, Phys. Rev. 133 (1964) B444

5) J. Bernstein and G. Feinberg, Phys. Rev. 125 (1962) 1741

6) W. Williamson and G. Salzman, Phys. Rev. Lett. 11 (1963) 224

7) A. C. T. Wu, T. T. Wu and K. Fuchel, Phys. Rev. Lett. 11 (1963) 390

8) S. M. Berman and Y. S. Tsai, Phys. Rev. Lett. 11 (1963) 483

9) N. Cabibbo and R. Gatto, Phys. Rev. 124 (1961) 1577

10) G. Feinberg and H. S. Mani, Phys. Rev. Lett. 11 (1963) 448

11) E. Ferrari and F. Selleri, Phys. Rev. Lett. 7 (1961) 381

12) S. D. Drell and K. Hiida, Phys. Rev. Lett. 7 (1961) 199

13) S. D. Drell, Revs. Mod. Phys. 33 (1961) 458

14) S. A. Bludman and J. A. Young, Phys. Rev. 126 (1962) 303;

J. A. Young and S. A. Bludman, Phys. Rev. 131 (1963) 2326

15) T. D. Lee and C. N. Yang, Phys. Rev. 128 (1962) 885 